\title{
Avoiding Burnouts in Clinical Practice
}

\section{Nabil F Bissada*}

Department of Periodontics, School of Dental Medicine, Case Western Reserve University, Cleveland, Ohio 44106-4905, USA

With the rapid changes and advancement in the management and treatment of our patients, many dentists are more susceptible than ever to early burnouts.

Dentistry is a profession of details and demands close attention. In addition, learning new treatments and how to use the new technologies in the modern dental office put more stress on the clinician that might be a reason for the increased prevalence of burnout. Being a burnout dentist has a significant impact on the doctor- patient relationship, as well as on the work environment in the dental office. The article published in this issue of Journal Dentistry on "Management and prevention of burnout in the dental practitioner" by Basson [1], describes early manifestations of burnout and suggests several ways for prevention and early treatment.This is an interesting article that can raise awareness of impending burnout among members of the dental team.

In another article by Elamrousy et al. [2], the authors reported on the high success rate of immediately restored implants placed immediately after extraction of periodontally compromised anterior teeth and suggested that this is a valid operative approach that leads to predictable outcome if adequate pre and post-operative measures are taken.

In the article published by Kolokitha et al. [3], where the authors reported on a rare case of hypodontia in a young boy with a history of Kowasaki disease stressed the importance of prophylaxis before initiation of dental treatment.

These three articles mentioned in this editorial comment are among several other interesting papers recently published in the Journal Dentistry. The information is of great clinical relevance and is applicable for better care and management of patients in our dental practices.

Dentists usually modify their approach to clinical care as time goes by. How do we determine that the changes we made are beneficial without adding more stress to the dental team? New knowledge is gained from different sources including reading professional journals. As health care professionals we have responsibility to our patients.

I trust that this issue of Journal Dentistry will be of great benefit to you as you strive to maintain a safe and healthy practice.

\section{References}

1. Basson RA (2013) Management and Prevention of Burnout in the Dental Practitioner. Dentistry 3: 168.

2. Elamrousy WA, Nassar M, Ragheb AM, Alnomany FA, Marzok MA (2013) Radiographic Bone Changes around Immediately Placed Immediately Restored Dental Implants in Periodontally Compromised Sites. Dentistry 3: 161.

3. Kolokitha OE, Chatzistavrou E, Almpani K (2013) A Rare Case of Hypodontia in Kawasaki Disease: Review of the Literature and Case Report. Dentistry 3: 164.

*Corresponding author: Nabil F Bissada, Professor and Chair, Department of Periodontics, School of Dental Medicine, Case Western Reserve University, 2124 Cornell Road, Cleveland, Ohio 44106-4905, USA, Tel: (216) 368-6752; Fax: (216) 368-3204; E-mail: nabil.bissada@case.edu

Received November 2, 2013; Accepted November 13, 2013; Published November 15, 2013

Citation: Bissada NF (2013) Avoiding Burnouts in Clinical Practice. Dentistry 3 e113. doi:10.4172/2161-1122.1000e113

Copyright: (c) 2013 Bissada NF. This is an open-access article distributed under the terms of the Creative Commons Attribution License, which permits unrestricted use, distribution, and reproduction in any medium, provided the original author and source are credited. 